\title{
Targeting peripheral $\kappa$-opioid receptors for the non-addictive treatment of pain
}

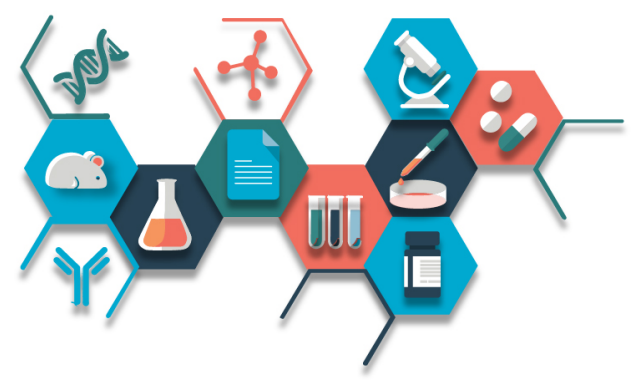

\author{
Tyler C Beck ${ }^{*, 1}$ \& Thomas A Dix ${ }^{1,2}$ \\ ${ }^{1}$ Department of Drug Discovery \& Biomedical Sciences, Medical University of South Carolina Campus, 280 Calhoun Street, PO Box \\ 250140, Charleston, SC 29424-2303, USA \\ 2JT Pharmaceuticals, Inc., 300 West Coleman Blvd, Suite 203, Mount Pleasant, SC 29464-2303, USA \\ *Author for correspondence: Tel.: +1 843876 5092; beckt@musc.edu
}

Drug addiction to prescription $\mu$-opioid agonists used in the setting of pain is a major public health threat, affecting millions of Americans. $\mathrm{k}$-opioid agonists (KOAs) may serve as a possible solution. KOAs have demonstrated indistinguishable analgesic activity relative to $\mu$-opioid agonists in models of acute and chronic pain; however, conventional KOAs suffer from central nervous system (CNS)-mediated psychoactive side-effects. In this review, we discuss our efforts, as well as other's efforts, in developing peripherally restricted KOAs with retained or improved efficacy in rodent models of pain. Results indicate that our lead compound, JT09, acts as efficacious as morphine in alleviating peripheral pain, while failing to produce undesired CNS-mediated side-effects. In this review, we discuss our former results and future directions.

First draft submitted: 30 May 2019; Accepted for publication: 26 June 2019; Published online:

2 September 2019

Keywords: analgesic • kappa • opioid • pain • peripheral

Chronic pain management is cited as the most common reason Americans seek medical intervention, affecting 20\% of the total population [1-4]. Current pain medications in use, which are largely $\mathrm{mu}(\mu)$ opioid receptor agonists (MOAs), are often ineffective in the treatment of moderate to severe chronic pain and have many other significant limitations associated with the side-effects of use. Side-effects associated with MOAs include nausea, vomiting, constipation, depressed breathing, renal toxicity, thromboembolic risk, and neurotoxicity [5]. Importantly, such compounds promote significant abuse liability and lead to the development of tolerance, thus requiring dose escalations to maintain therapeutic efficacy. In 2015, there were over 42,000 reported accidental opioid overdose deaths in the USA, which is a $600 \%$ increase since 1999 [6,7]. These statistics outline the significance of the opioid epidemic and the need for a non-addictive analgesic.

Despite the current opioid crisis, prescription of opioids continues to remain strikingly high due to the lack of development of novel classes of analgesics. To illustrate this, in 2004, the peptide ziconotide and the small molecule pregabalin were the first novel pain agents approved for use in the USA in over 40 years [8]. Both compounds function by targeting presynaptic N-type voltage-gated calcium channels (N-VGCCs) to inhibit neurotransmitter release at the synapse and thus blocking pain sensation; however, both have significant limitations. The administration of ziconotide requires an invasive procedure, in which a pump is implanted in the patient's spinal cord, which significantly limits its utility. Pregabalin has been approved for only a limited number of indications, such as peripheral neuropathy and postherpetic neuralgia. Both ziconotide and pregabalin demonstrate significant toxicities due to their selectivity for calcium channels, such as nausea, vomiting and neuropsychiatric disturbances. Despite their extensive side-effect profile, both drugs continue to demonstrate financial success, as ziconotide and pregabalin achieve $\$ 200$ million/year and $\$ 2.7$ billion/year in revenue, respectively. Pregabalin has been a top 10 prescribed' drug for many years. Additionally, the centrally acting opioid analgesic Tapentadol was introduced in 2009 [9]. Tapentadol demonstrates potent analgesic activity utilizing a dual mode of action as a MOA and as a norepinephrine reuptake inhibitor; however, similar to conventional MOAs, Tapentadol is associated with abuse liability and respiratory depression. Despite its limitations, 2019 revenue for Tapentadol is estimated to be between $\$ 200$ and 210 million [10]. All told, these figures illustrate the potential economic impact and benefit that a novel class of analgesics would provide. 
There have been recent efforts to develop novel non-addictive analgesics to be used in the clinical management of chronic pain. Of note, Stefanucci et al. have developed mixed opioid agonist/N-VGCC blocker peptides for the treatment of pain. Furthermore, MORs and N-VGCCs are co-localized on pain-modulating neurons in the CNS [11]. The development of a multi-target molecule combining an opioid pharmacore and an N-VACC blocker may provide synergistic activity and could serve as a therapeutic candidate for the treatment of severe chronic pain. Following in vitro and in vivo testing, compound $5 \mathrm{a}$ emerged as a lead compound for future development. Compound $5 \mathrm{a}$ demonstrated the best binding affinity for the MOR, while simultaneously blocking co-localized N-VACCs in the CNS. The analgesic potency of compound $5 \mathrm{a}$ was significant; however, the compound must be administered intrathecally, thus limiting its potential applications. Additional limitations may include adverse effects associated with N-VACC blockage in the CNS and agonism of centrally located $\mu$-opioid receptors (MORs), as previously described; however, preliminary results warrant further modification and investigation of these multitarget hybrid compounds. Additionally, there have been recent attempts to synthesize nociceptin variants for the treatment of acute and chronic pain [12]. The nociceptin/orphanin FQ (N/OFQ) receptor, also known as the $\kappa$-type 3 opioid receptor, is a member of the opioid receptor family and is widely distributed throughout the nervous system [13]. Nociceptin is an endogenous ligand that shares a degree of homology with dynorphin A, an endogenous KOA; however, nociceptin displays poor affinity for classical opioid receptors, while binding to the N/OFQ receptor with high affinity. N/OFQ receptor agonists promote analgesic activity with limited abuse liability, making such compounds attractive for future development for the treatment of pain and related disorders [14]. However, there is currently a lack of orally available N/OFQ receptor agonists, limiting their development as a class. Future studies focusing on formulation strategies to improve the oral bioavailability of nociception-derivatives are thus warranted.

$\kappa$-opioid agonists (KOAs) may serve as a promising approach to treating acute and chronic pain. KOAs demonstrate similar potency and efficacy in comparison to MOAs in models of visceral pain by acting on peripherally located $\kappa$ opioid receptors (KORs); however, KOAs also act on centrally located KORs, promoting untoward side effects, such as dysphoria, hallucinations, sedation and psychosis [15]. Thus, the development of pathway-biased KOAs or KOAs with limited CNS-penetration could serve as novel nonaddictive analgesics. Cara Therapeutics (CT, USA) compound CR665 (Figure 1A), has demonstrated a peripheral-selectivity of > 500-fold, with significant analgesic activity in models of acute and chronic; however, CR665 is not sufficiently orally bio-available, limiting its use as an out-patient pharmaceutical for chronic pain. The JT Pharmaceutical (SC, USA) compound JT09 (Figure 1B), a second-generation compound related to CR665, is an orally active peptide analgesic that is currently being studied for the indication of chronic pain. The peripherally restricted KOA, CR845 (Figure 1C), is currently under phase three clinical trials and was found to attenuate pruritus, as well as acute and chronic pain. The drug is being dosed intravenously (iv.) and was well tolerated in 300 patients during Phase II clinical trials [6]. Another established intravenously available KOA that acts primarily in the periphery is ICI 204,448 which has been shown to attenuate pruritus and inflammatory pain. Additionally, when administered to rats $10 \mathrm{~min}$ prior to the onset of myocardial ischemia, ICI 204,448 substantially reduced infarct size, demonstrating the potential use of KOAs for the indication of myocardial infarction [16]. However, ICI 204,448 demonstrated sedative effects at doses as low as $2 \mathrm{mg} / \mathrm{kg}$ iv., which could be explained by peripheral activation of the vagus nerve or due to centrally penetrative effects on the cerebellum [2].

In this review, we describe the characteristics of JT09 that demonstrate its potential for development as a novel, broad-spectrum and analgesic.

\section{Methods \& results}

Modification of the position 4 D-Arg residue of CR665 yielded derivatives with enhanced stability and oral availability $[17,18]$. Lead compound JT09 demonstrated improved pharmacokinetic/pharmacodynamic (PK/PD) properties (Table 1) [2]. These PK/PD numbers are minimal estimates, since $\mu$ - or $\delta$-activities were not detected. It was also demonstrated that JT09 does not act as a KORs antagonist or an inverse agonist.

In rat pain efficacy models, JT09 was evaluated in an acetic acid writhing assay, an established measure of peripherally mediated pain, and in a hot plate assay, a measure of centrally mediated pain. In the acetic acid writhing assay, rats were orally gavaged (po.) with JT09 $(20 \mathrm{mg} / \mathrm{kg}$ ), while control rats received either morphine $(10 \mathrm{mg} / \mathrm{kg}$, intraperitoneally [ip.]) or saline $(0.2 \mathrm{ml}$, po.). JT09 demonstrated indistinguishable efficacy when compared with morphine in the acetic acid writhing assay for peripheral pain. In the hot plate model, rats received JT09 (20 mg/kg, po.), while control rats received morphine ( $10 \mathrm{mg} / \mathrm{kg}$, ip.). Rats were tested for centrally mediated pain 20 min later by measuring the latency to paw licking behavior following placement on a $53^{\circ} \mathrm{C}$ hot plate. In 
(A)

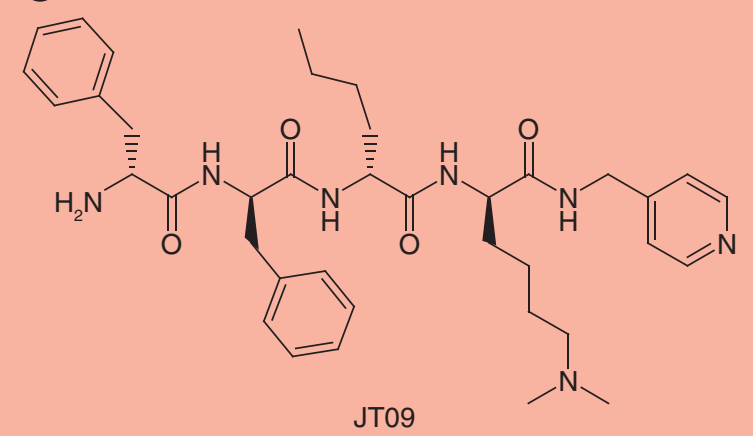

(B)

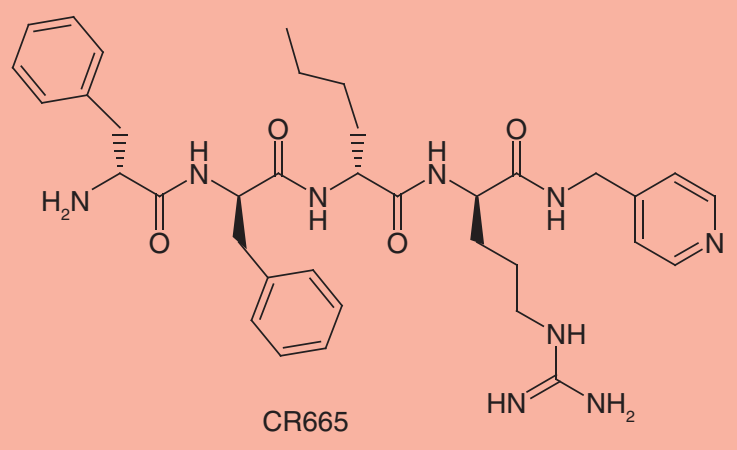

(C)

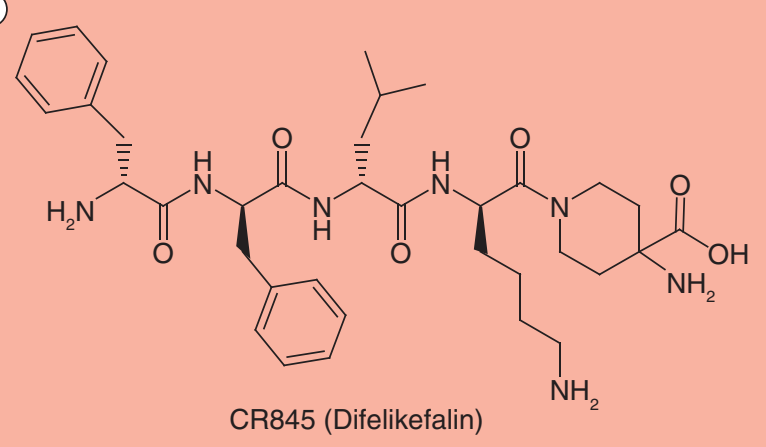

Figure 1. Chemical structures of JT09 (1A), CR665 (1B) and CR845 (1C).

Table 1. Preliminary biological data for JT09.

\begin{tabular}{|ll|}
\hline Biological parameter & Results \\
\hline Peripheral selectivity & $>938$-fold \\
\hline$\kappa$ over $\mu$ or $\delta$ selectivity & $>33,400$-fold \\
\hline Oral $\mathrm{ED}_{50}$ & $4.7 \mathrm{mg} / \mathrm{kg}$ \\
\hline Intravenous $\mathrm{ED}_{50}$ & $0.032 \mathrm{mg} / \mathrm{kg}$ \\
\hline ED: Effective dose. & \\
\hline
\end{tabular}

the hot plate model, JT09 did not attenuate centrally mediated pain, thus supporting that JT09 does not cross the blood-brain barrier.

Behavioral assays were performed in order to assess the side effect profile associated with JT09. Addiction is a major issue associated with conventional analgesics, such as morphine and other opioid derivatives. In order to assess the abuse liability associated with JT09, rats were studied in a self-administration model, in which rats were trained to press a lever to receive an iiv. drug infusion. Rats failed to maintain lever responding when switched from sucrose to JT09 (20 mg/kg/infusion), extinguishing lever pressing behavior within 5 days. This finding suggests that JT09 does not promote reward seeking behavior. When switched to cocaine ( $50 \mu \mathrm{g} / \mathrm{ul} /$ infusion), rats resumed lever pressing behavior, thus proving that the rats tested were not deficient in reward processing. In order to further assess abuse liability, a conditioned place preference model was performed, where rats would receive alternate oral doses of JT09 $(20 \mathrm{mg} / \mathrm{kg})$ and saline $(0.2 \mathrm{ml})$ in their respective compartment (right or left, randomly assigned). The amount of time spent in each particular compartment was used to determine if the rats developed an association of JT09 with a particular compartment, which is a sign of addictive predilection. Results demonstrated that rats did not develop a preference for JT09 over saline, further suggesting that JT09 does not promote drug seeking behavior. 
KOAs are commonly discontinued due to the induction of psychoactive side-effects, such as dysphoria and sedation [19]. In order to assess the induction of dysphoria, a forced swim assay was performed. Depressive effects are measured by the amount of time spent immobile during the last 4 min of each trial. Administration of JT09 $(20 \mathrm{mg} / \mathrm{kg}$, po.) did not promote immobility beyond that of saline, whereas salvinorin A ( $1 \mathrm{mg} / \mathrm{kg}$, ip.), a centrally acting KOA, promoted significant immobility [5]. This finding suggests that JT09 does not promote dysphoria. In order to assess sedation, rats were studied in locomotor boxes to assess activity levels following the administration of either saline $(0.2 \mathrm{ml}$, po.), morphine $(10 \mathrm{mg} / \mathrm{kg}$, ip.) or JT09 $(20 \mathrm{mg} / \mathrm{kg}$, po.). Results indicated that there was a significant difference in the activity level of rats that received morphine when compared with JT09; however, the activity levels were indistinguishable when comparing rats that received JT09 and saline. This supports that JT09 does not promote sedative effects due to its high peripheral selectivity for the $\kappa$ receptor over the $\mu$ receptor. JT09 was assessed in a multiple dose study, where rats were dosed at their $\mathrm{EC}_{50}$ for 14 days, sacrificed and then assessed via histological examination. On examination, rats administered JT09 did not demonstrate any gross or microscopic changes to the sampled tissues. This suggests that JT09 does not promote any noticeable toxicity in rats at the ED90 dose level. Further toxicity studies will be performed administering JT09 at higher dose levels.

\section{Significance}

There are many pharmacological and non-pharmacological treatments of chronic pain; however, very few treatment modalities have proven to demonstrate sustainable efficacy. In particular, MOAs provide sufficient analgesia acutely in many patients, yet suffer from untoward side effects, such as those mentioned above. Given the significant burden of current opioid and non-opioid medications used in pain management, there remains a significant need for a novel class of analgesics that lacks abuse liability and has a diminished side effect profile. Much like the wellknown MOAs, KOAs have significant analgesic properties; however, KOAs potentiate negative side effects such as sedation, hallucinations and dysphoria, thus limiting their development as a viable therapeutic. Developing a KOA with limited CNS penetration would provide analgesic coverage against chronic pain without the aforementioned negative side effects associated with the agonism of centrally located KORs. Pre-clinical and clinical studies show that peptide-derived, peripherally restricted, orally active KOAs such as JT09, CR845 and ICI 204,448 offer promising qualities and could potentially be the solution to this problem. Further, application of the JT Pharma peptide modification strategy to CR665 imparted oral bioavailability, while enhancing the potency and PK/PD properties of the parent compound. CR845, a derivative of CR665 under development by Cara Therapeutics, demonstrated $15 \%$ oral bioavailability in Phase I clinical trials following reformulation to improve oral delivery. Given our preliminary results in preclinical studies, we suspect that JT09 will demonstrate significantly improved oral bioavailability without the specific need for reformulation. Additionally, JT09 does not promote sedation, whereas IC 204,448 demonstrated significant depression of locomotor activity [20].

On average, 115 people a day die from an opioid-induced drug overdose, thus emphasizing the potential groundbreaking nature of developing a nonaddictive analgesic with retained efficacy [7]. In comparison, 44 people died per day during the peak of the HIV/AIDs crisis, further outlining the significance of this public health epidemic. A possible solution to this issue is the peripherally restricted KOA, JT09. The oral EC $_{50}$ of JT09 in rats appears to be druggable when extrapolated to human doses, while retaining the efficacy of morphine. Further, we have established that JT09 does not promote CNS-mediated effects associated with centrally mediated KOAs or commonly used opioids such as morphine (i.e., sedation, dysphoria, tolerance, addiction, etc.). Thus, we propose that JT09 has potential for development as a novel analgesic.

\section{Future perspective \& steps}

The described research findings reveal many areas of future development for JT09 and related compounds. Our laboratory plans to perform long-term toxicological studies, as well as absorption, distribution, metabolism and excretion (ADME) studies. Additionally, we plan to assess the efficacy of JT09 in rat models of chronic pain. As previously stated, there is an urgent need for new therapeutics for the treatment of chronic pain and peripherally restricted KOAs as a class may serve as non-addictive alternatives to conventional opioid analgesics.

A full report of this letter is in press at the European Journal of Pharmacology (PMID: 31103632). 


\section{Executive summary}

- The use of $\mu$-opioid receptor agonists in the management of acute and chronic pain is considered the standard of care in most of the world.

- Despite their potent analgesic properties, $\mu$-opioid receptor agonists promote negative central nervous system mediated side-effects, the most notable being abuse liability.

- The development of nonaddictive analgesics for the treatment of acute and chronic pain is a major unmet need and could help combat the current opioid epidemic.

- $\kappa$-opioid receptor agonists demonstrate similar analgesic activity in comparison to $\mu$-opioid agonists, while lacking abuse liability, thus making the $\kappa$-opioid receptor an attractive target for drug development.

- Our laboratory has developed a novel, peripherally restricted, orally active $\kappa$-opioid agonist that acts as efficacious as morphine in alleviating pain and is devoid of the negative side-effects associated with agonism of centrally located opioid receptors.

\section{Acknowledgments}

The authors are grateful for the contributions by CM Reichel, SM Ghee, SS Bhadsavle and ML Kopfman, for helping conduct experiments; PM Woster and IR Kumarasinghe for synthesizing experimental compounds; and KL Helke for performing histological studies.

Financial \& competing interests disclosure

This work was supported by NIH grants DA-036398, NS-090629 and MH-65099 to TA Dix and a grant from SCE\&G to TC Beck. TC Beck does not declare a conflict of interest. TA Dix is the Chief Scientific Officer of JT Pharmaceuticals, Inc. All experiments and data analysis described in this review were performed at the Medical University of South Carolina, independent of JT Pharmaceuticals, Inc. The authors approve deposition of the final peer-reviewed manuscript to the NIH Manuscript Submission in compliance with the NIH Public Access Policy. The authors have no other relevant affiliations or financial involvement with any organization or entity with a financial interest in or financial conflict with the subject matter or materials discussed in the manuscript apart from those disclosed.

No writing assistance was utilized in the production of this manuscript.

\section{Open access}

This work is licensed under the Attribution-NonCommercial-NoDerivatives 4.0 Unported License. To view a copy of this license, visit http://creativecommons.org/licenses/by-nc-nd/4.0/

\section{References}

Papers of special note have been highlighted as: $\bullet$ of interest; $\bullet \bullet$ of considerable interest

1. American Society of Addiction Medicine. Opioid addiction 2016 facts \& figures (2019). www.asam.org/docs/default-source/advocacy/opioid-addiction-disease-facts-figures.pdf

2. Beck TC, Reichel CM, Ghee SM, Helke KL, Bhadsavle SS, Dix TA. Non-addictive orally-active kappa opioid agonist for the treatment of peripheral pain in rats. Eur. J. Pharmacol. 856, 172396 (2019).

-. The original research article presenting the data described in this review article.

3. DeHaven-Hudkins DL, Dolle RE. Peripheral restricted opioid agonists and novel analgesic agents. Curr. Pharm. Design 10(7), 743-757 (2004).

- The fundamental paper that discusses the significance of peripherally restricted $\kappa$-opioid agonists.

4. Gaskin DJ, Richard P. Appendix C: the economic costs of pain in the United States In: Relieving Pain in America: A Blueprint for Transforming Prevention, Care, Education, and Research. Institute of Medicine. National Academic Press, DC, USA, 1-22 (2011).

5. Goldberg D, McGee S. Pain as a Global Health Priority. BMC Public Health 11(1), 770 (2011).

6. Cara Therapeutics (2019). www.caratherapeutics.com/

7. Centers for Disease Control and Prevention. Understanding the epidemic (2017). www.cdc.gov/drugoverdose/epidemic/index.html

8. Hughes FM Jr., Shaner BE, Brower JO, Woods RJ, Dix TA. Develoment of a peptide-derived orally available kappa-opioid agonist targeting peripheral pain. Open Med. Chem. J. 7, 16-22 (2013).

-. The original research article discussing the first generation screen of novel, orally active, peripherally restricted $\mathrm{k}$-opioid agonists.

9. Thompson CA. Tapentadol approved as pain reliever. Am. J. Health Syst. Pharm. 66(1), 8 (2009). 
10. Corporate News, Guidance, Management Comments Collegium Pharma (COLL) announces FY19 product revenue Guidance for Xtampza ER and Nucynta (2019). www.streetinsider.com/Corporate+News/Collegium+Pharma+\%28COLL\%29+Announces $+\mathrm{FY} 19+\mathrm{Pr}$ oduct+Revenue+Guidance+for+Xtampza+ER+and+Nucynta/14980511.html

11. Setfanucci A, Novellino E, Macedonio G et al. Design, synthesis and biological profile of mixed opioid agonist/N-VGCC blocker peptides. New J. Chem. 42(8), 5656-5659 (2018).

12. Erdei AI, Borbely A, Magyar A et al. Biochemical and pharmacological investigation of novel nociception/OFQ analogues and N/OFQ-RYYRIK hybrid peptides. Peptides 112, 106-113 (2018).

13. Mollereau C, Parmentier M, Mailleux P et al. ORL1, a novel member of the opioid receptor family. Cloning, functional expression and localization. FEBS Lett. 341(1), 33-38 (1994).

14. Lin AP, Ko M-P. The therapeutic potential of nociceptin/orphanin FQ receptor agonists as analgesics without abuse liability. ACS Chem. Neurosci. 4(2), 214-224 (2013).

15. MacPherson RD. The pharmacological basis of contemporary pain management. Pharmacol. Ther. 88(2), 163-185 (2000).

16. Peart JN, Gross ER, Gross GJ. Effect of exogenous kappa-opioid receptor activation in rat model of myocardial infarction. J. Cardiovasc. Pharmacol. 43(3), 410-415 (2004).

17. Lundquist JT, Dix TA. Asymmetric synthesis of [omega]-bromo-2(S)-azido acids as precursors for the synthesis of novel amino acids. Tetrahedron Lett. 39(8), 775-778 (1998).

- The original research article discussing the design of the non-natural amino acid analogs discussed in this review article.

18. Riviere PJ-M. Peripheral kappa-opioid agonists for visceral pain. Br. J. Pharmacol. 141(8), 1331-1334 (2004).

- The fundamental paper that discusses the use of $\kappa$-opioid agonists in visceral pain.

19. Stein SC, Clark JD, Oh U et al. Peripheral mechanisms of pain and analgesia. Brain Res. Rev. 60(1), 90-113 (2008).

- The fundamental paper discussing the peripheral mechanisms of pain and analgesia referenced in this review article.

20. Kumar N. Kappa opioid receptor agonists: new targets in treatment of pain. Victoria University of Wellington, Victoria, NZ (2014). http://hdl.handle.net/10063/4963 